\title{
Spondyloarthritis patients with and without intestinal symptoms - searching for discriminating biomarkers
}

\author{
EWA KONTNY', JOANNA DMOWSKA-CHALABA \\ ${ }^{1}$ Department of Pathophysiology and Immunology, National Institute of Geriatrics, Rheumatology and Rehabilitation, Warsaw, Poland \\ ${ }^{2}$ Early Arthritis Clinic, National Institute of Geriatrics, Rheumatology, and Rehabilitation, Warsaw, Poland
}

\begin{abstract}
Spondyloarthritis (SpA) is often complicated with subclinical gut inflammation. This study was aimed at searching for biomarkers discriminating SpA patients with and without intestinal symptoms. A group of 29 SpA patients and 33 healthy volunteers (control) were included in the study. Based on clinical evaluation, the patient cohort was subdivided into two groups: 1) SpA accompanied by various intestinal symptoms suggesting gut inflammation (group 2, $n=14$ ) and 2) without such complications (group 1 , $n=15)$. Serum concentrations of interleukins (IL) $(I L-10, I L-17 A / F, I L-22, I L-23)$, tumour necrosis factor (TNF), bone-homeostasis-related factors (osteoprotegerin-OPG and Dickkopf-1 - DKK-1), and the concentrations of selected gut inflammation-associated factors (intestinal fatty acid binding protein-iFABP, claudin $3-C L D N 3$ and calprotectin) in samples of sera and/or urine or stool, respectively, were measured by specific ELISA. Serum concentrations of tested factors were similar in SpA patients and control. Faecal calprotectin level was higher in patients but did not discriminate between group 1 and 2. Compared to group 1, group 2 was characterized by elevated erythrocyte sedimentation rate (ESR), higher serum CLDN3 and DKK-1 levels. In SpA patients, serum DKK-1 concentrations correlated with systemic inflammation markers $(R=0.6, p<0.01)$, while serum CLDN3 was found to be an independent risk factor $(O R=4.5, p=0.021)$ for the occurrence of intestinal symptoms. We conclude that in SpA patients, up-regulated circulating levels of CLDN3 seem to be related to intestinal complication, while the quantity of circulating DKK-1 reflects the intensity of systemic inflammation.
\end{abstract}

Key words: cytokines, spondyloarthritis, gut inflammation, claudin 3.

(Centr Eur J Immunol 2019; 44 (4): 414-422)

\section{Introduction}

Spondyloarthritis (SpA) comprises a group of inter-related inflammatory diseases that share a genetic background and have overlapping clinical features [1]. Ankylosing spondylitis (AS) and psoriatic arthritis (PsA) represent the main members of this group. Bacterial infections of the gastrointestinal or genitourinary tracts are relevant triggers of $\mathrm{SpA}$, and, importantly, intracellular bacteria may spread the infection to other anatomical locations [2]. Inflammation develops in various joints of the body (spinal, sacroiliac, peripheral), paraspinal tissues, and entheses. Erosive bone damage is associated with pathological bone remodelling manifested by bone spur and bridge formation leading to severe disability [3]. Extra-articular manifestations, especially intestinal inflammation, are common in SpA. In 40-60\% of AS and $46 \%$ of early SpA patients gut inflammation, verified by endoscopic and histological examination, is present, and $5-10 \%$ of them eventually develop inflammatory bowel disease (IBD), more often Crohn's disease than ulcerative colitis [4-6]. The aetiopathogenesis of IBD in SpA is poorly understood. It is suggested that aberrant homeostasis of gut-associated lymphoid tissue (GALT), abnormalities in the composition of gut microbiota, and/or loss of tolerance to its physiological components may critically contribute to the development of this complication [7-9]. In addition, medications used to treat inflammatory joint disease, especially nonsteroidal anti-inflammatory drugs (NSAIDs), may adversely affect the bowel disease and trigger drug-induced enteropathy [10]. Accumulating evidence point to the bowel as an active player in the immunological processes typical of $\mathrm{SpA}$, and there is a need to search for useful biomarkers and predictors of intestinal symptoms in this disease [9].

We hypothesised that the immune status and the levels of gut inflammation biomarkers in biological material might discriminate between SpA patients with and with-

Correspondence: Ewa Kontny, Department of Pathophysiology and Immunology, National Institute of Geriatrics, Rheumatology, and Rehabilitation, 1 Spartańska St., 02-637 Warsaw, Poland, e-mail: ewa.kontny@wp.pl Submitted: 27.10.2017; Accepted: 5.02.2018 
out intestinal symptoms. To verify this assumption, the concentrations of: 1) various cytokines implicated in SpA pathogenesis (i.e. in inflammation and aberrant bone homeostasis) [3, 9] and 2) select proteins, intestinal fatty acid binding protein (iFABP), claudin 3 (CLDN3), and calprotectin (CALP), as proposed biomarkers of gut inflammation in acute and chronic pathological conditions [11, 12], were measured in biological material (sera, urine, stool) of the above patient cohort and control healthy volunteers.

\section{Material and methods}

\section{Patients and controls}

In this cross-sectional study, 29 patients with the diagnosis of SpA according to the Assessment of SpondyloArthritis International Society (ASAS) criteria [13] and 33 healthy volunteers of similar age were included in the study (Table 1). Although there was a higher proportion of men among the patients than among healthy volunteers,

Table 1. Characteristics of the study groups*

\begin{tabular}{|c|c|c|c|}
\hline Characteristics & $\begin{array}{l}\text { Healthy volunteers } \\
\qquad(n=33)\end{array}$ & $\begin{array}{c}\text { SpA } \\
(n=29)\end{array}$ & $p$-value \\
\hline \multicolumn{4}{|l|}{ Demographics } \\
\hline Age, years & $39.5(26-58)$ & $42(26-62)$ & 0.34 \\
\hline Sex, female (F) / male (M), $n$ & $23 \mathrm{~F} / 10 \mathrm{M}$ & $12 \mathrm{~F} / 17 \mathrm{M}$ & NA \\
\hline Disease duration, years & & $2.0(0-20)$ & NA \\
\hline Disease activity, (BASDAI), score & & $6.3(3.6-8.1)$ & NA \\
\hline low $(<4), \%$ & & 10 & NA \\
\hline moderate $(\geq 4-<6), \%$ & & 29 & NA \\
\hline high $(\geq 6), \%$ & & 61 & NA \\
\hline Laboratory values & & & NA \\
\hline $\mathrm{ESR}, \mathrm{mm} / \mathrm{h}$ & & $19(5-105)$ & NA \\
\hline $\mathrm{CRP}, \mathrm{mg} / \mathrm{l}$ & & $22(6-84)$ & NA \\
\hline Serum concentrations & & & NA \\
\hline IL-10, pg/ml & $4.6(0-20)$ & $5(0-21)$ & 0.32 \\
\hline IL-17 A/F, pg/ml & $0(0-373)$ & $21(0-949)$ & 0.34 \\
\hline $\mathrm{IL}-22, \mathrm{pg} / \mathrm{ml}$ & $37.3(12-193)$ & $38(0-444)$ & 0.73 \\
\hline $\mathrm{IL}-23, \mathrm{pg} / \mathrm{ml}$ & $0(0-926)$ & $0(0-2012)$ & 0.72 \\
\hline $\mathrm{TNF}, \mathrm{pg} / \mathrm{ml}$ & $4.4(0-184)$ & $0(0-429)$ & 0.76 \\
\hline $\mathrm{OPG}, \mathrm{pg} / \mathrm{ml}$ & 1035 (619-2544) & $908(327-4418)$ & 0.59 \\
\hline DKK-1, pg/ml & $4175(3140-7104)$ & $4444(1907-6564)$ & 0.22 \\
\hline DKK-1/OPG, ratio & $4.16(1.33-7.84)$ & $4.44(0.52-11.46)$ & 0.63 \\
\hline $\mathrm{iFABP}, \mathrm{pg} / \mathrm{ml}$ & $256(47-954.7)$ & $248.6(107-970.3)$ & 0.78 \\
\hline \multicolumn{4}{|l|}{ CLDN3 } \\
\hline serum concentration, $\mathrm{ng} / \mathrm{ml}$ & $25(18.4-40)$ & $23.4(15.4-40)$ & 0.66 \\
\hline urine concentration, $\mathrm{ng} / \mathrm{ml}$ & $0.323(0-1.04)$ & $0(0-123.4)$ & 0.09 \\
\hline \multicolumn{4}{|l|}{ Calprotectin } \\
\hline faecal concentration, $\mathrm{mg} / \mathrm{kg}$ & $3.2(0.17-13.8)$ & $18.9(0.39-391.7)$ & 0.00017 \\
\hline Medications, $\%$ & & & NA \\
\hline NSAIDs & & 100 & NA \\
\hline Non-biologic DMARDs & & 44.5 & NA \\
\hline
\end{tabular}

${ }^{*}$ Except where indicated otherwise, values are the median (range); SpA - spondyloarthritis, BASDAI - the Bath Ankylosing Spondylitis Disease Activity Index, ESR - erythrocyte sedimentation rate, CRP - C-reactive protein, IL - interleukin, TNF - tumour necrosis factor, OPG - osteoprotegerin, DKK-1 - Dickkopf 1 , iFABP - intestinal fatty acid binding protein, CLDN3 - claudin3, NSAIDs - non-steroidal anti-inflammatory drugs, DMARDs - disease-modifying anti-rheumatic drugs, NA - not applicable 
no significant differences in tested parameters were found between males and females in both groups (not shown). The participants were recruited from patients admitted to the Early Arthritis Diagnostic Department of the National Institute of Geriatrics, Rheumatology, and Rehabilitation (NIGRR) or NIGRR staff, respectively. Before enrolment, written informed consent was obtained from all participants. This study meets all criteria contained in the Declaration of Helsinki and was approved by the Ethics Committee of the NIGRR. The majority of patients suffered from AS $(55 \%)$ or axial SpA $(21 \%)$, while other forms of SpA were less frequent (14\% of PsA and 10\% of undifferentiated SpA). Disease activity was assessed using Bath Ankylosing Spondylitis Disease Activity Index (BASDAI). All patients received NSAIDs, and almost half of them were treated with non-biological disease modifying anti-inflammatory drugs (DMARDs), either sulfasalazine $(35 \%)$ or methotrexate $(10 \%)$ (Table 1). Based on complete clinical evaluation and medical history, SpA patients were classified into two groups. Group $1(n=15)$ comprised patients without any intestinal symptoms, while group $2(n=14)$ included patients who reported intestinal symptoms, such as recurrent diarrhoea, abdominal pain and cramping, and blood or mucous in stool. However, intestinal symptoms were not verified by endoscopic examination. Samples of peripheral blood, urine, and stool were collected in the morning. Serum was isolated by routine laboratory methods, urine was centrifuged for $20 \mathrm{~min}$ at $1000 \mathrm{~g}$, and supernatant was collected. After preparation, samples of serum, urine, and stool were stored in aliquot at $-70^{\circ} \mathrm{C}$ until assayed. Before testing, the faecal extracts were prepared using an extraction device (CALPRO AS, Norway) and according to the manufacturer's description.

\section{Enzyme-linked immunosorbent assay (ELISA)}

The concentrations of tested factors in collected samples were measured using commercially available ELISA kits specific for: 1) interleukin (IL)-10, IL-17 A/F, IL-23, and tumour necrosis factor (TNF) (all from eBioScience, an Affymetrix Company, San Diego, CA, USA), 2) IL-22 (Abcam, Cambridge, UK), 3) dickkopf-1 (DKK-1) (R\&D Systems, Minneapolis, MN, USA), 4) calprotectin (CALPRO AS, Norway), 5) CLDN 3 (My BioSource, Inc., San Diego, CA, USA), and 6) iFABP (Hycult Biotech, Uden, the Netherlands). The above assays were performed according to the manufacturer's protocols. The ELISA for osteoprotegerin (OPG) was done using recombinant human OPG as a standard and antibodies specific for human OPG (all from R\&D Systems). Briefly, mouse monoclonal antibody was used as the capture antibody while goat polyclonal biotinylated antibody was applied as the detection antibody. Streptavidin-peroxidase conjugate (Jackson ImmunoResearch Laboratories, West Grove, PA, USA) followed by $o$-phenylenediamine dihydrochloride (OPD) (Sigma, ) was added to develop the enzymatic reaction.
The concentrations of CALP were determined in faecal samples, CLDN3 in both sera and urine samples, and others in sera samples only.

\section{Statistical analysis}

Data were analysed using Statistica 10 software (StatSoft Inc., OK, USA). The Mann-Whitney $U$ test was used for intergroup comparison. Correlation was assessed using Spearman's rank test ( $R$ value is shown). Univariate logistic regression analysis was used to calculate the odds ratio (OR) and identify risk factor(s) for intestinal symptoms. To perform this analysis, the independent predictor variable was split into categories based on quartiles. The $p$-values $<0.05$ were considered significant.

\section{Results}

General characteristics, and laboratory and immune parameters of the study population are shown in Table 1.

\section{Faecal concentration of calprotectin discriminates between spondyloarthritis patients and healthy volunteers}

As shown in Table 1, there were no significant differences in serum concentrations of tested cytokines between the total SpA patient group and healthy volunteers. Among the gut inflammation-associated biomarkers, only faecal concentrations of CALP were significantly up-regulated in SpA patients, while the levels of serum iFABP as well as serum and urine CLDN3 were similar both in SpA patients and healthy donors.

\section{Spondyloarthritis patients with intestinal symptoms are characterised by more pronounced systemic inflammation and raised serum DKK-1 concentration}

A comparison of demographic and clinical characteristics of SpA patient groups with and without intestinal symptoms failed to disclose any significant differences between them in age, sex, disease duration, disease activity, and medications. Also, serum concentrations of pro-inflammatory cytokines (TNF, interleukins of IL-17/IL-23 axis) and anti-inflammatory IL-10 were comparable in both patient groups (Table 2). However, for the SpA group with intestinal symptoms a slightly higher level of systemic inflammation was characteristic, as indicated by elevation of ESR (Table 2, Fig. 1), as well as less pronounced differences in CRP (Table 2) values. In addition, in this patient group serum DKK-1 (but not OPG) and consequently the DKK-1/OPG ratio were significantly higher than in patients without gut involvement (Fig. 1, Table 2). Similar significant differences were noted when the SpA patients 
Table 2. Comparative characteristics of the spondyloarthritis patient subgroups*

\begin{tabular}{|c|c|c|c|}
\hline Characteristics & $\begin{array}{c}\text { SpA } \\
\text { without intestinal symptoms } \\
(n=15)\end{array}$ & $\begin{array}{c}\mathrm{SpA} \\
\begin{array}{c}\text { with intestinal symptoms } \\
(n=14)\end{array} \\
\end{array}$ & $p$-value \\
\hline \multicolumn{4}{|l|}{ Demographics } \\
\hline Age, years & $44(27-62)$ & $42(26-61)$ & 0.55 \\
\hline Sex, female (F) / male (M), $n$ & $7 \mathrm{~F} / 8 \mathrm{M}$ & $5 \mathrm{~F} / 9 \mathrm{M}$ & \\
\hline Disease duration, years & $2(0.5-20)$ & $2.5(0.5-15)$ & 0.54 \\
\hline Disease activity (BASDAI) & $6.2(3.6-7.4)$ & $6.3(4.3-8.1)$ & 0.69 \\
\hline Sacroiliitis (rtg) & $1.5(1-4)$ & $1.5(0-4)$ & 0.45 \\
\hline \multicolumn{4}{|l|}{ Laboratory values } \\
\hline $\mathrm{ESR}, \mathrm{mm} / \mathrm{h}$ & $15(5-50)$ & $37(10-105)$ & 0.049 \\
\hline $\mathrm{CRP}, \mathrm{mg} / \mathrm{l}$ & $21(6-43)$ & $29.5(12-84)$ & 0.15 \\
\hline \multicolumn{4}{|l|}{ Serum cytokine concentrations } \\
\hline IL-10, pg/ml & $5.2(0-21)$ & $5.4(1.2-10.4)$ & 0.55 \\
\hline IL-17 A/F, pg/ml & $0(0-949)$ & $60.6(0-739)$ & 0.16 \\
\hline IL-22, pg/ml & $36.6(0-256)$ & $40.7(0-444)$ & 0.25 \\
\hline IL-23, pg/ml & $0(0-1074)$ & $0(0-2012)$ & 0.38 \\
\hline $\mathrm{OPG}, \mathrm{pg} / \mathrm{ml}$ & $927(737-4125)$ & 907 (327-4418) & 0.40 \\
\hline $\mathrm{TNF}, \mathrm{pg} / \mathrm{ml}$ & $10.3(0-429)$ & $0(0-87)$ & 0.60 \\
\hline \multicolumn{4}{|l|}{ Medications, $\%$} \\
\hline NSAIDs & 100 & 100 & \\
\hline Non-biologic DMARDs & 40 & 50 & \\
\hline
\end{tabular}

with intestinal symptoms were compared to healthy volunteers (for serum DKK-1 and DKK-1/OPG ratio $p$ values = 0.001 and 0.04 , respectively; data not shown). Interestingly, as depicted in Figure 2, in the total SpA group serum DKK-1 concentrations positively and strongly correlated with the systemic inflammation markers (ESR and CRP). In addition, there was positive but rather moderate correlation between serum levels of DKK-1 and pro-inflammatory IL-17 A/F. Moreover, DKK-1 turned out to be rather poorly associated with the presence of intestinal symptoms $(\mathrm{OR}=1.001$; Table 3$)$. Thus, it is likely that up-regulation of DKK-1 in the SpA group with intestinal symptoms is a consequence of the higher level of systemic inflammatory response characteristic for these patients.

\section{Higher concentration of serum claudin 3 in spondyloarthritis patients is associated with the presence of intestinal symptoms}

Comparison of both patient groups regarding the levels of gut inflammation-associated biomarkers revealed significant elevation of serum CLDN3 in SpA patients with intestinal symptoms (Fig. 1). Importantly, serum concen- trations of CLDN3 turned out to be an independent association factor for intestinal symptoms in SpA patients. Based on the regression analysis, we found that although in the total $\mathrm{SpA}$ group this factor only slightly elevated the risk of intestinal disorders $(\mathrm{OR}=1.125)$, every quantitative increase of serum CLDN3 from one to the next quartile category was associated with several-fold higher $(\mathrm{OR}=$ 4.5 ) risk of this complication (Table 3 ). In addition, serum concentrations of CLDN3 positively, albeit rather weakly, correlated with the number of intestinal symptoms (Fig. 2). As shown in Figure 1, there was also a trend towards elevation of serum iFABP, but the differences did not reach statistical significance $(p=0.1)$. By contrast, the faecal concentration of CALP was similar in both patient groups.

\section{Discussion}

The present study failed to show significant differences in serum concentrations of tested cytokines between the total group of SpA patients and healthy volunteers. The possible explanations are clinical heterogeneity and limited sample size of the patient group (Table 1). Cytokines of IL-17/IL-23 axis have essential homeostatic functions 

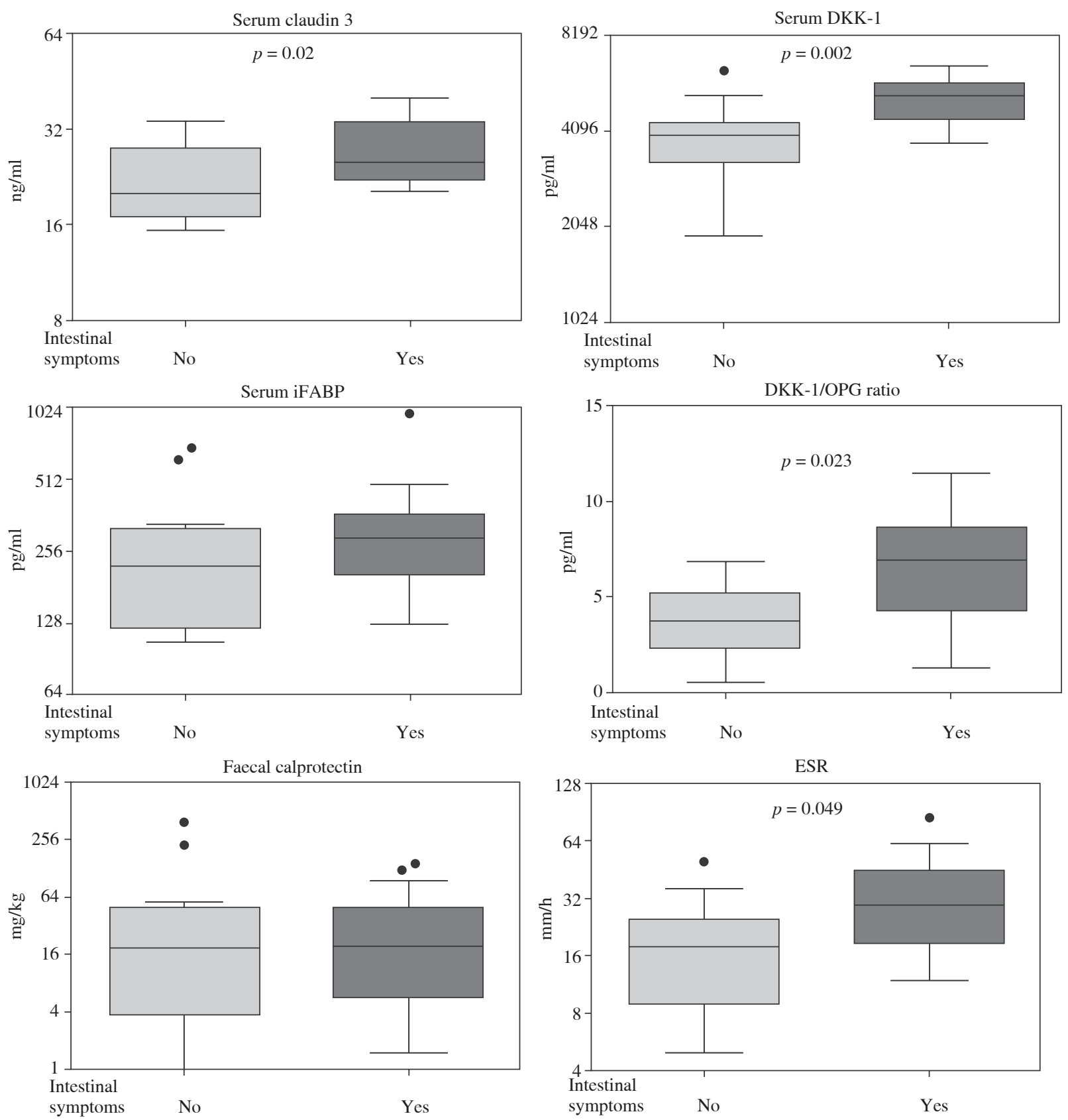

Fig. 1. Comparison of spondyloarthritis patients with and without intestinal symptoms. Results are expressed as the median (horizontal line) with interquartile range (IQR, box), lower and upper whiskers (data within $3 / 2 \times \mathrm{IQR}$ ), and outliers (points) (Tukey's box). iFABP - intestinal fatty acid binding protein, DKK-1 - Dickkopf 1, OPG - osteoprotegerin, ESR - erythrocyte sedimentation rate. For statistically significant differences between patient groups $p$ values are shown

both in the joint-associated tissues and in the gut, and they are thought to play an important role in the pathogenesis of SpA $[9,14]$. However, their overexpression in inflamed tissues (intestine, entheses, synovial tissues and fluids), an inconsistency in quantitative assessment of their serum levels (higher than or the same as in healthy controls), and conflicting results when searching for an association between their circulating pool and clinical data (e.g. exis- tence or lack of correlation with disease activity) point to these cytokines acting primarily in restricted anatomical locations [15-19].

From among tested biomarkers of gut-inflammation only the faecal concentration of CALP differentiated between SpA patients and healthy control (Table 1). Calprotectin is a heterodimer formed by S100A8 and S100A9 proteins, produced at the site of inflammation by activated 

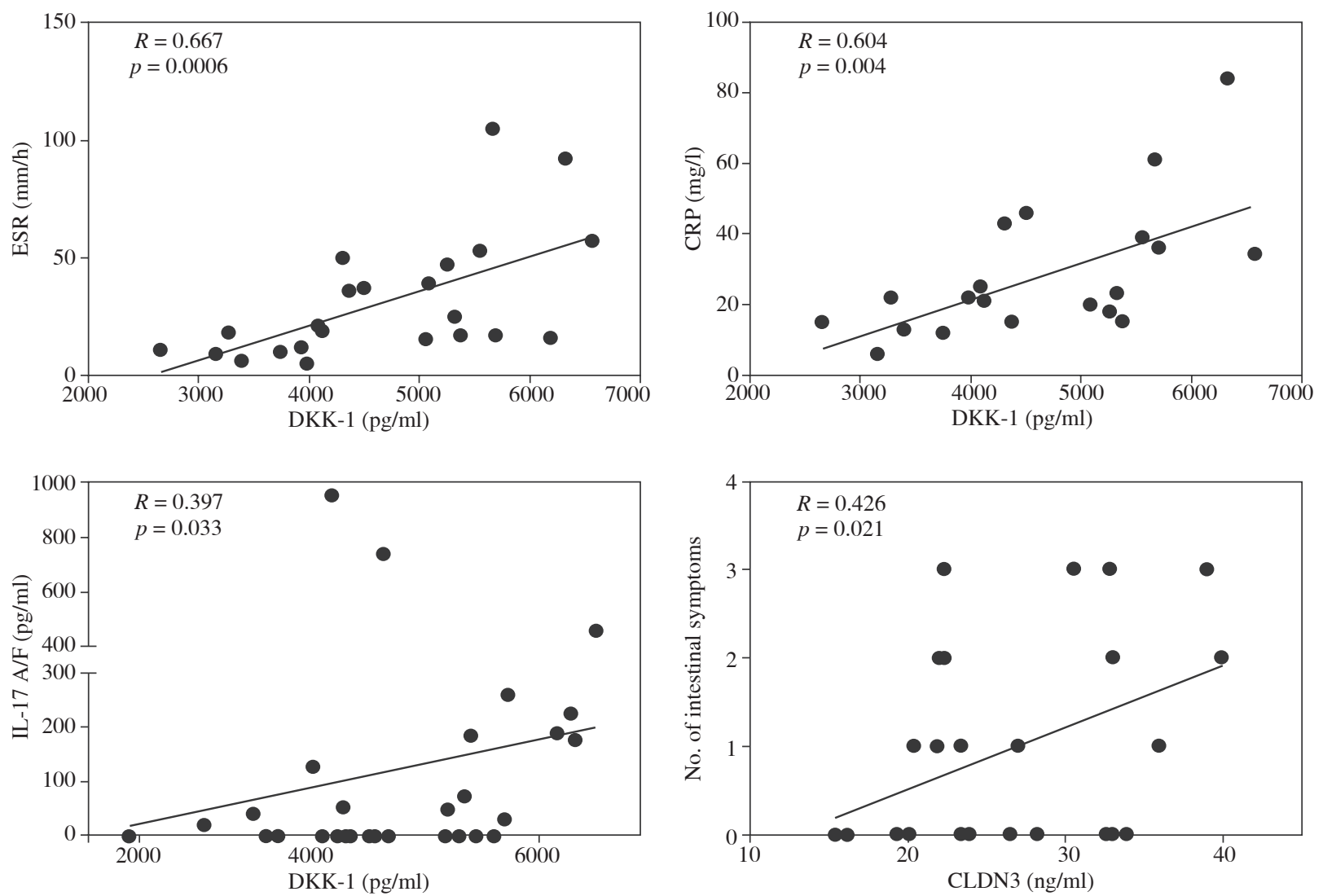

Fig. 2. Relationship between the serum Dickkopf 1 (DKK-1) and claudin 3 (CLDN3) concentrations and clinical or laboratory data. Each point represents one patient. The correlation was assessed using Spearman's rank test; $R$ and $p$ values are shown. Note that although Spearman's rank correlations were performed, the regression lines were used for graphic purposes only. ESR - erythrocyte sedimentation rate, CRP - C-reactive protein, IL-17 A/F - interleukin $17 \mathrm{~A} / \mathrm{F}$

Table 3. Potential association factors for intestinal symptoms in SpA patients

\begin{tabular}{|c|c|c|c|}
\hline \multicolumn{2}{|l|}{ Factor } & \multirow[t]{2}{*}{ OR $(95 \% \mathrm{CI})$} & \multirow[t]{2}{*}{$p$-value } \\
\hline Name & Quartile category (range) & & \\
\hline \multirow[t]{4}{*}{ CLDN3 } & All $(15.4-40 \mathrm{ng} / \mathrm{ml})$ & $1.125(0.996-1.271)$ & 0.058 \\
\hline & $\mathrm{I}(<20 \mathrm{ng} / \mathrm{ml})$ & $4.5(1.256-16.12)$ & 0.021 \\
\hline & II $(>20-30 \mathrm{ng} / \mathrm{ml})$ & & \\
\hline & III $(>30$ ng/ml $)$ & & \\
\hline DKK-1 & All (1907.4-6564.4 pg/ml) & $1.001(1.000331-1.002388)$ & 0.01 \\
\hline
\end{tabular}

leukocytes (monocytes and neutrophils) during their interaction with activated endothelium. Elevated circulating CALP concentrations, often correlated with laboratory variables of inflammation, clinical disease activity, and treatment response, have been found in numerous chronic inflammatory diseases, including rheumatic diseases (RD) and IBD [20-22]. Faecal CALP is an unspecific marker of gut inflammation, reflecting the level of intestinal neutrophil infiltration [23]. In SpA patients it was reported to be associated with age, acute phase reactants, disease duration, and clinical activity but not with gastrointestinal symptoms [24]. Recently, however, elevated faecal CALP has been proposed as a biomarker of SpA patients with concomitant bowel inflammation and the main predictor 
of IBD development [25-27]. Unfortunately, in the present study, despite up-regulation of faecal CALP in SpA patients compared to the healthy controls, we failed to find such association. Because all our patients were treated with NSAIDs, it is likely that these drugs triggered CALP release from immune cells and thus elevated this protein concentration in stool samples (Table 1). Moreover, when we used the manufacturer's recommended cut-off value for positivity $(50 \mathrm{mg} / \mathrm{kg})$, stool samples of none of healthy volunteers and only six SpA patients $(20.7 \%)$ were positive for faecal CALP. Of these only three patients reported intestinal symptoms, and therefore truly CALP-positive patients were included into both SpA subgroups. Consequently, we failed to show significant differences in faecal CALP levels between SpA patients with and without intestinal symptoms (Fig. 1). Despite the limitations of our categorisation method (not supported by an endoscopic evaluation), these patient subgroups differed in the ESR value, serum DKK-1, and claudin 3 concentrations, which were significantly higher in SpA patients with intestinal symptoms (Fig. 1).

Dickkopf-1, an inhibitor of osteoblastogenesis and master regulator of bone homeostasis, shifts the balance toward new bone formation when its expression is decreased, or toward bone erosion when it is overexpressed [28]. In $\mathrm{SpA}$, a prototype bone-forming disease, assessment of serum DKK-1 levels has yielded conflicting results. A recent study that included a large cohort $(>700)$ of early axial SpA patients has given convincing support of significant serum DKK-1 increase compared to healthy controls [29]. Despite overexpression, this inhibitor is dysfunctional and thus facilitates pathological rebuilding of bone in SpA [30]. Intriguingly, in patients with axial SpA the degree of sacroiliac joint inflammation was reported to be linked to gut inflammation [31]. Moreover, accumulating evidence shows that in these patients, serum DKK-1 concentrations are related not only to syndesmophyte formation but also to systemic inflammation $[29,32]$. The present results, showing positive correlation between serum DKK-1 levels and classical biomarkers of systemic inflammation (ESR, CRP), are consistent with these observations (Fig. 2). In addition, we found significant but weaker association between serum DKK-1 and IL-17 A/F levels (Fig. 2). This, in turn, is in line with known function of proinflammatory cytokines, including IL-17 and TNF, which, at least in vitro, work in synergy as potent triggers of DKK-1 production [33]. As mentioned before, in SpA cytokines of the IL-17/IL-23 axis are thought to act primarily in specific anatomical locations and thus are not sensitive systemic inflammation markers [15-19]. This explains both the lack of difference in IL-17 A/F serum levels between patients with and without intestinal symptoms and the weaker association of this cytokine with DKK-1 observed in our study. Altogether, the above results suggest that elevation of serum DKK-1 in SpA patients with intestinal symptoms reflects a higher level of systemic inflammation than direct association with the presence of gut complications (Fig. 2, Table 3).

Importantly, the present results point to serum CLDN3 as an independent risk factor for intestinal symptoms, distinguishing SpA with gut involvement from SpA without this complication, which also correlates with the number of intestinal symptoms (Table 3, Figs. 1 and 2). Claudins are essential components of epithelial tight junctions (TJs). TJs regulate the flux of ions and solutes, and maintain the integrity of epithelial barrier. In the intestine all epithelial cells are joined in this manner. Claudins function as "leaky" pore-forming or "tight" pore-sealing proteins. Claudin-3 is a "tight" protein that plays a barrier function. It is found in many different epithelia including the gastrointestinal tract where it is highly expressed, especially in the colon [34-36]. In the intestinal inflammatory disorders abnormalities of claudins expression, that modify epithelial homeostasis and barrier function, have been observed. In active ulcerative colitis expression of CLDN3 in the gut is either unaffected or reduced and is accompanied by an increase of its urinary concentrations [35, 36]. Similarly, in Crohn's and celiac diseases reduced intestinal CLDN3 expression has been reported to be associated with alterations in TJ ultrastructure, which normalise under treatment $[36,37]$. Based on data from both experimental and clinical settings, it is suggested that measurement of urinary CLDN3 by Western blotting technique can be used as a non-invasive marker for intestinal TJ loss [38]. We applied the ELISA technique for this purpose. In contrast to the above data concerning IBD patients, we found comparable and negligible amounts of CLDN3 in urine but quite high concentrations of this protein in the sera of both healthy volunteers and SpA patients (Table 1). Based on the urine CLDN3 levels, it is tempting to speculate that our SpA patients have generally preserved function of intestinal TJs. However, our finding of significantly higher serum concentrations of CLDN3 in SpA patients with intestinal symptoms than in those without such complication questions the above supposition (Fig. 1). Accumulating evidence shows that the dysregulation of the claudin circulating pool occurs in different pathological conditions, suggesting that proteins of this family might represent potential blood-borne biomarkers of diagnostic and/or prognostic value [39-41]. For example, the presence of CLDN3 in exosomes released by prostate cancer cells and elevated plasma CLDN3 concentrations in men suffering from prostate cancer have recently been reported [42]. Moreover, plasma CLDN3 has been proposed as a putative prognostic biomarker associated with more aggressive prostate cancer, predictive of disease progression. The evaluation of circulating claudins in SpA patients has not been done so far. Because CLDN3 is expressed in different epithelia, it is probable that not only impaired gut epithelia supply the circulating pool in our SpA patients. However, our further 
observations suggest that this is not the case because we found that serum CLDN3 is not only an independent risk factor for intestinal symptoms (Table 3) but also correlates with the intensity of this complication (Fig. 2). Our observation is in accordance with a recent report showing that bacterial ileitis and damage of intestinal mucosal barrier, present in the majority (70\%) of AS patients, are associated with increased blood levels of zonulin, a protein that modulates the permeability of epithelial TJs of the digestive tract [43]. In addition, we observed a trend for the elevation of serum iFABP levels in SpA patients with intestinal symptoms compared to patients without any signs of gut involvement (Fig. 1). Intestinal FABP is a small (14-15 kDa) protein, which in humans is expressed solely in enterocytes of intestinal epithelium, more of the villus than of the crypts, constituting up to $2 \%$ of cytoplasmic content of these cells. It is present throughout the small intestine, and its highest expression is in the jejunum. The physiological role of iFABP is to take up fatty acids from the lumen of the intestine and to traffic them within enterocytes to organelles $[44,45]$. The level of circulating iFABP in healthy individuals is low, reflecting normal turnover of enterocytes, but upon loss of membrane integrity of these cells iFABP is released into the circulation [45]. Because of its low molecular weight, iFABP, similarly to CLDN3, can pass through the glomerular filter and arise in the urine. Significantly elevated plasma/serum and urine concentrations of iFABP have been found in patients with gut-related pathology, e.g. in intestinal ischaemia, celiac disease, ulcerative colitis, or necrotising enterocolitis [4548]. Therefore, the measurement of both circulating and urine iFABP levels is thought to represent a non-invasive method for assessment of early intestinal damage in various pathological conditions characterised by continuous, villus mucosal damage that does not extend to the muscle layers $[11,12,48]$.

\section{Conclusions}

We report for the first time that SpA patients with intestinal symptoms are characterised by more intense systemic inflammation, accompanied by elevation of circulating DKK-1, an inhibitor of osteoclastogenesis, as well as by up-regulated serum level of CLDN3, an essential component of epithelial TJs. Moreover, in SpA patients we found serum CLDN3 to be an independent risk factor for intestinal symptoms. Based on the known biological role of DKK-1 and CLDN3, it can be assumed that in SpA patients with reported intestinal symptoms the bone homeostasis is more disturbed and that there is discrete damage of intestinal epithelium. However, due to the small sample size and the subjective method of intestinal symptom evaluation, the present results should be regarded as preliminary.

The authors declare no conflict of interest.

\section{References}

1. Paramarta JE, Baeten D (2014): Spondyloarthritis: from unifying concepts to improved treatment. Rheumatology (Oxford) 59: 1547-1559.

2. Costello ME, Elewaut D, Kenna TJ, Brown MA (2013): Microbes, the gut and ankylosing spondylitis. Arthritis Res Ther 15: 214 .

3. Kontny E (2014): New aspects of spondyloarthritis pathogenesis. Part III - arthritis, pathological bone remodeling. Reumatologia 52: 247-254.

4. Rudwaleit M (2006): Ankylosing spondylitis and bowel disease. Best Pract Clin Rheumatol 20: 451-471.

5. Stolwijk C, van Tubergen A, Castillo-Ortiz JD, Boonen A (2015): Prevalence of extra-articular manifestation in patients with ankylosing spondylitis: a systematic review and meta-analysis. Ann Rheum Dis 74: 65-73.

6. Van Praet L, Van den Bosch FE, Jacques P, et al. (2013): Microscopic gut inflammation in axial spondyloarthritis: a multiparametric predictive model. Ann Rheum Dis 72: 414-417.

7. Rosenbaum JT, Davey MP (2011): Hypothesis: time for a gut check: HLA-B27 predisposes to ankylosing spondylitis by altering the microbiome. Arthritis Rheum 63: 3195-3198. doi: 10.1002/ar.30558.

8. Diamanti AP, Rosado MM, Laganá B, D’Amelio R (2016): Microbiota and chronic inflammatory arthritis: an interwoven link. J Transl Med 14: 233.

9. Wendling D (2016): The gut in spondyloarthritis. Joint Bone Spine 83: 401-405.

10. Orlando A, Renna S, Perricone G, Cottone M (2009): Gastrointestinal lesions associated with spondyloarthropathies. World J Gastroenterol 15: 2443-2448.

11. Wells JM, Brummer RJ, Derrien M, et al. (2017): Homeostasis of the gut barrier and potential biomarkers. Am J Physiol Gastointest Liver Physiol 312: G171-G193.

12. Grootjans J, Thuijls G, Verdam F, et al. (2010): Non-invasive assessment of barrier integrity and function of the human gut. World J Gastointest Surg 2: 61-69.

13. Rudwaleit M, van der Heijde D, Landewé E, et al. (2011): The Assessment of SpondyloArthritis International Society for classification criteria for peripheral spondyloarthritis and for spondyloarthritis in general. Ann Rheum Dis 70: 25-31.

14. Miossec P (2017): Update in interleukin-17: a role in the pathogenesis of inflammatory arthritis and implication for clinical practice. RMD Open 3, e000284.

15. Ciccia F, Bombardieri M, Principato A, et al. (2009): Overexpression of interleukin-23, but not interleukin-17, as an immunologic signature of subclinical intestinal inflammation in ankylosing spondylitis. Arthritis Rheum 60: 955-965.

16. Mei Y, Pan F, Gao J, et al. (2011): Increased serum IL-17 and IL-23 in the patients with ankylosing spondylitis. Clin Rheumatol 30: 269-273.

17. Appel H, Maier R, Wu P, et al. (2011): Analysis of IL-17(+) cells in facet joints of patients with spondyloarthritis suggests that the innate immune pathway might be of greater relevance than the Th-17-mediated adaptive immune response. Arthritis Res Ther 13: R95.

18. Chen WS, Chang YS, Lin KC, et al. (2012): Association of serum interleukin-17 and interleukin-23 levels with disease activity in Chinese patients with ankylosing spondylitis. J Chin Med Assoc 75: 303-308.

19. Muntean L, Lungu A, Gheorghe SR, et al. (2016): Elevated serum levels of sclerostin are associated with high disease activity and functional impairment in patients with axial spondyloarthritis. Clin Lab 62: 589-597. 
20. Boyapati RK, Rossi AG, Satsangi J, Ho GT (2016): Gut mucosal DAMPs in IBD: from mechanisms to therapeutic implications. Mucosal Immunol 9: 567-582.

21. Ometto F, Friso L, Astorri D, et al. (2017): Calprotectin in rheumatic diseases. Exp Biol Med (Maywood) 242: 859-873.

22. Turina MC, Yeremenko N, Paramarta JE, et al. (2014): Calprotectin (S100A8/9) as serum biomarker for clinical response in proof-of-concept trials in axial and peripheral spondyloarthritis. Arthritis Res Ther 2014, 16: 413.

23. Tibble J, Teahon K, Thjodleifsson B, et al. (2000): A simple method for assessing intestinal inflammation in Crohn's disease. Gut 47: 506-513.

24. Prajzlerová K, Grobelná K, Pavelka K, et al. (2016): An update on biomarkers in axial spondyloarthritis. Autoimmunity Rev 15: 501-509.

25. Cypers H, Varkas G, Beeckman S, et al. (2016): Elevated calprotectin levels reveal bowel inflammation in spondyloarthritis. Ann Rheum Dis 75: 1357-1362.

26. Klinberg E, Strid H, Ståhl A, et al. (2017): A longitudinal study of fecal calprotectin and the development of inflammatory bowel disease in ankylosing spondylitis. Arth Res Ther 19: 21.

27. Østgard RD, Deleuran BW, Dam MY, et al. (2017): Faecal calprotectin detects subclinical bowel inflammation and may predict treatment response in spondyloarthritis. Scand J Rheumatol Jun 26: 1-8. doi: 10.1080/03009742.2017.1299216.

28. Klavdianou K, Liossis SN, Sakkas L, Daoussis D (2017): The role of Dickkopf-1 in joint remodeling and fibrosis: A link connecting spondyloarthritis and scleroderma? Semin Arthritis Rheum 46: 430-438.

29. Nocturne G, Pavy S, Boudaoud S, et al. (2015): Increase in Dickkopf-1 serum level in recent spondyloarthritis. Data from the DESIR cohort. PLoS One 10: e0134974.

30. Daoussis D, Liossis SN, Solomou EE, et al. (2010): Evidence that Dkk-1 is dysfunctional in ankylosisg spondylitis. Arthritis Rheum 62: 150-158.

31. Van Praet L, Jans L, Carron P, et al. (2014): Degree of bone marrow oedema in sacroiliac joint of patients with axial spondyloarthritis is linked to gut inflammation and male sex: results from GIANT cohort. Ann Rheum Dis 73: 1186-1189.

32. Sakellariou GT, Iliopoulos A, Konsta M, et al. (2017): Serum levels of Dkk-1, sclerostin and VEGF in patients with ankylosing spondylitis and their association with smoking, and clinical, inflammatory and radiographic parameters. Join Bone Spine 84: 309-315.

33. Shaw AT, Maeda Y, Gravallese EM (2016): IL-17A deficiency promotes periosteal bone formation in a model of inflammatory arthritis. Arthritis Res Ther 18: 104.

34. Capaldo CT, Powell DN, Kalman D (2017): Layered defense: how mucus and tight junctions seal the intestinal barrier. J Mol Med 95: 927-934.

35. Garcia-Hernandez V, Quiros M, Nusrat A (2017) : Intestinal epithelial claudins: expression and regulation in homeostasis and inflammation. Ann NY Acad Sci 1397: 66-79.

36. Barmeyer C, Fromm M, Schultzke JD (2017): Active and passive involvement of claudins in the pathophysiology of intestinal inflammatory diseases. Pflugers Arch - Eur J Physiol 469: 15-26.

37. Goswami P, Das P, Verma AK, et al. (2014): Are alterations of tight junctions at molecular and ultrastructural level different in duodenal biopsies of patients with celiac disease and Crohn's disease? Virchows Archiv 465: 521-530.

38. Thuijls G, Derikx JP, de Haan JJ, et al. (2010:) Urine-based detection of intestinal tight junction loss. J Clin Gastroenterol 44: e14-e19.
39. Karabulut M, Alis H, Bas K, et al. (2015): Clinical significance of serum claudin-1 and claudin-7 levels in patients with colorectal cancer. Mol Clin Oncol 3: 1255-1267.

40. Tas F, Bilgin E, Erturk K, Duranyildiz D (2016): Clinical significance of serum claudin-1 levels in melanoma patients. Melanoma Res 26: 377-381.

41. Yücel M, Kotan D, Gurol Çiftçi G, et al. (2016): Serum levels of endocan, claudin-5 and cytokines in migraine. Eur Rev Med Pharmacol Sci 20: 930-936.

42. Worst TS, von Hardenberg J, Gross JC, et al. (2017): Database-augmented mass spectrometry analysis of exosomes identifies claudin 3 as a putative prostate cancer biomarker. Mol Cell Proteomics 16: 998-1008.

43. Ciccia F, Guggino G, Rizzo A, et al. (2017): Dysbiosis and zonulin upregulation alter gut epithelial and vascular barriers in patients with ankylosing spondylitis. Ann Rheum Dis 76: 1123-1132.

44. Gajda AM, Storch J (2015): Enterocyte fatty acid binding proteins (FABPs): different functions of liver- and intestinal-FABPs in the intestine. Prostaglandins Leukot Essent Fatty Acids 93: 9-16.

45. Lieberman JM, Sacchettini J, Marks C, Marks WH (1997): Human intestinal fatty acid binding protein: report of an assay with studies in normal volunteers and intestinal ischemia. Surgery 121: 335-342.

46. Derikx JP, Vreugdenhil AC, Van den Neucker AM, et al. (2009): A pilot study on the noninvasive evaluation of intestinal damage in celiac disease using I-FABP and L-FABP. J Clin Gastroenterol 43: 727-733.

47. Wiercinska-Drapalo A, Jaroszewicz J, Siwak E, et al. (2007): Intestinal fatty binding protein (I-FABP) as a possible biomarker of ileitis in patients with ulcerative colitis. Regul Pept 147: 25-28.

48. Evennett NJ, Hall NJ, Pierro A, Eaton S (2010): Urinary intestinal fatty acid-binding protein concentration predict extend of disease in necrotizing enterocolitis. J Pediatr Surg 45: 735-740. 